\title{
FORMULATION AND EVALUATION OF LIQUISOLID COMPACT OF CHLORTHALIDONE
}

\author{
Geeta K Patel ${ }^{\star}$ and Parth D Patel \\ Department of Pharmaceutics, S. K. Patel College of Pharmaceutical Education \\ and Research, Ganpat University, Kherva-384012, Gujarat, India.
}

\begin{abstract}
Liquisolid compacts were used to formulate water insoluble drugs in non-volatile solvents and converting into acceptably flowing and compressible powders. The main objective of present investigation was to enhance the dissolution rate of poorly water soluble drug Chlorthalidone by using liquisolid technique. Several liquisolid tablets were prepared by using different carrier materials such as microcrystalline cellulose (Avicel pH-102) and coating material such as Aerosil respectively. Polyethylene glycol 400 was used as nonvolatile water miscible liquid vehicle. The liquid loading factors for such liquid vehicle was calculated to obtain the optimum amounts of carrier and coating materials necessary to produce acceptable flowing and compactable powder admixtures viable to produce compacts. Before compression, powdered mass were evaluated for various parameters like flow properties, content uniformity etc. All the prepared formulations were compressed using $13 \mathrm{~mm}$ punch after addition of $5 \%$ Sodium starch glycolate and crospovidone to each formulation. The formulated liquisolid tablets were evaluated for post compression parameters such as weight variation, hardness, drug content uniformity,percentage friability and disintegration time. The in-vitro release characteristics of the pure drug, drug from marketed tablets (as reference) and liquisolid technique (test sample), were studied. DSC study was performed to check drug excipients compatibility. The spectra revealed that there was no interaction between drug and excipients.The results showed that liquisolid formulations of Chlorthalidone exhibited higher percentage of drug release than marketed formulation.
\end{abstract}

Keywords: Chlorthalidone, Sodium starch glycolate and crospovidone.

\section{INTRODUCTION}

Solubility is one of the most important physicochemical properties of any drug because lowsolubility can affect the bioavailability of orally administered dosage forms. Thus, it is veryimportant to enhance the solubility of a poorly soluble drug. For absorption, a drug must bepresent in the form of an aqueous solution at the site of absorption. Poorly water-soluble drugs involve many difficulties in the development of pharmaceutical dosage forms for oral delivery systems due to their low bioavailability. It has been established that the active ingredient in a solid dosage form must undergo dissolution before it is available for absorption from the gastro intestinal tract. ${ }^{1}$

The dissolution properties of a drug and its release from a dosage form have a basic impact on its bioavailability. ${ }^{2}$ Solving solubility problems is a major challenge for the pharmaceutical industry with development of new pharmaceutical products, since nearly half of the active substances being identifiedthrough the new paradigm in highthroughput screening are either insoluble or poorly soluble in water. ${ }^{3,4}$

Nearly one-third of drugs in development arewater insoluble and one-half fail in trials because of underprivileged pharmacokinetics. ${ }^{5}$ These poorly water soluble drugs are 
alliedwith slow drug absorption leading toinadequate and variable bioavailability andgastrointestinal mucosal toxicity of drugs. ${ }^{6}$ Various methods employed to improve the dissolution characteristics ofpoorly water soluble drugs are solubilization, $\mathrm{pH}$ adjustment, cosolvancy, microemulsion, self emulsification, polymeric modification, drug complexation, and micronization, use of surfactant as a solubilizing agent, the prodrug approach and solid dispersion. The new technique developed by Spireas 'liquisolid system' is the most promising method for improving the dissolution properties of poorly solubledrugs. ${ }^{7}$

A liquisolid system refers to formulations formed by conversion of liquiddrugs, drug suspensions or drug solution innon-volatile solvents, into dry, non-adherent,free- flowing and compressible powdermixtures by blending the suspension orsolution with selected carriers and coatingmaterials. ${ }^{3}$ Drug presentin the liquid medicament in liquisolid system is in the solubilised or molecularly dispersedstate, so the dissolution can be enhanced by increased surface area and betterwetting properties. $^{8}$ The technique of liquisolid compacts has been successfullyemployed to improve the in vitro release of poorly water soluble drugssuch as carbamazepine, Atorvastatin calcium, famotidine, Piroxicam, Indomethacin, Rofecoxib, Carvedilol, Irbesartan etc. The advantages of Liquisolid techniques includes simplicity, low cost and capability of industrialproduction. ${ }^{9-16}$

Chlorthalidone is used to treat high blood pressure and fluid retention caused by various conditions, including heart disease. Chlorthalidone is a phthalamide derivative of benzene sulphonamide and is designated as 2- chloro-5-(1-hydroxy-3-oxo-1-isoindolinyl) benzene sulphanilamide. Chlorthalidone is widely accepted for its excellent antihypertensive and anti-diuretic effect.Chlorthalidone is considered first-line therapy for management of uncomplicated hypertension as there is strong evidence from meta-analyses that thiazide diuretics such as chlorthalidone reduce the risk of stroke, myocardial infarction, heart failure, and cardiovascular all-cause mortality in patients with hypertension. ${ }^{18}$ Chlorthalidone is BCS IV drug which is poorly soluble in water $(0.986 \mathrm{mg} / \mathrm{L})$. It is rapidly absorbed after oral administration with peak plasma concentration at 30 to $60 \mathrm{~min}$.

\section{MATERIALS AND METHODS}

Chlorthalidone and microcrystalline cellulose (AvicelPH 102)were received as gift samples from Medley Pharmaceutical Ltd., Daman.
Aerosil 200 was obtained as gift sample from Yarrow Chem Products, Mumbai. Sodiam starch glycolate, Crospovidone, Mannitol,PEG 400, 300 and 600, Tween 20, Tween 80 were purchased from S D Fine Chem. Products, Mumbai. All other excipients and reagents used were of pharmaceutical grade.

\section{PREFORMULATION STUDY \\ Drug excipients compatibility (FTIR)}

Fourier transform infrared spectroscopy was employed to characterize further the possible interactions between the drug and the formulation excipients by the conventional $\mathrm{KBr}$ pellet method. The spectra were scanned over a frequency range $4000-400 \mathrm{~cm}^{-1}$ with a resolution of $4 \mathrm{~cm}^{-1}$.

\section{Solubility studies ${ }^{17}$}

The saturation solubility studies were carried out in different non volatile solvents shown in Table-1 i.e. PEG 400, PEG 600, Propylene Glycol (PG), Tween 20, Tween 80 , so as to select the best non volatile solvent for preparation of liquid medication. In brief, excess amount of Chlorthalidone was mixed with non-volatile solvents separately in $50 \mathrm{ml}$ vials. The mixtures were shaken on shaker for 48 hours. Then solutions were filtered through $0.45 \mu$ membrane filter and diluted suitably with $1 \%$ sodium lauryl sulphate (SLS) and analyzed UV spectrophotometrically (UV 1700, Shimadzu, Japan) at $275 \mathrm{~nm}$ for their drug content. Three determinations were carried out for each sample to calculate the solubility of Chlorthalidone.

\section{Measurement of Angle of Slide ${ }^{18}$}

This experiment was designed to measure the flowable liquid retention potential ( $\varphi$-value) for Avicel PH 102 (carrier material) and Aerosil (coating material) and the optimum liquid load factor. The $\varphi$-value of a powder is the maximum amount of given non-volatile liquid that can be retained inside powder bulk (w/w) while maintaining acceptable flowability, whereas is the mass ratio $(\mathrm{w} / \mathrm{w})$ of the liquid medication to the carrier powder in the liquisolid formulation. Powder admixtures containing $5 \mathrm{~g}$ of either carrier or coating with increasing quantity of nonvolatile liquid vehicle were mixed using a mortar and pestle. Each admixture was then placed on a shiny metal plate; the plate was then tilted until the admixture slides. The angle formed between the plate and the horizontal surface, at which admixture slides were measured as angle of slide $(\theta)$. The flowable liquid retention potential was calculated using the following equation: 


$$
\varphi \text {-Value }=\frac{\text { Weight of nonvolatile liquid }}{\text { Weight of carrier or coat }}
$$

Each admixture has specific $\varphi$-values which were determinedand plotted against respective measured angle of slide for allnonvolatile liquid vehicles. The $\varphi$-value that corresponds toan angle of slide of $33^{\circ}$ was reported to represent the flowableliquid retention potentials of powder admixtures.

\section{Preparation of Preliminary batch formulation}

The appropriate amounts of carrier and coatingmaterials used for each formulation depend upon $L f$ of thatformulation (Table-2). The liquid load factor (Lf) is calculated by using following formula

$$
\begin{aligned}
& \mathrm{Lf}=\phi+\varnothing(1 / R) \\
& \mathrm{Lf}=\mathrm{W} / \mathrm{Q} \\
& \mathrm{R}=\mathrm{Q} / \mathrm{q}
\end{aligned}
$$

Where, $\phi$ and $\varnothing$ are the values of the carrier and the coating powders respectively while $R$ is excipients ratio.

Liquisolid tablets of Chlorthalidone were prepared using composition mentioned in Table-3. Chlorthalidone and non-volatile solvent were accurately weighed in $25 \mathrm{ml}$ glass beaker and then sonicated until homogenous solution was obtained. The medication was incorporated into calculated quantity of carrier and coating material so liquisolid blend was obtained. The liquisolid powder was further blended with mannitol and $5 \%$ SSG and Crospovidone for $5 \mathrm{~min}$ and $1 \%$ magnesium stearate and Talc were added in the polybag just before compression. Liquisolid compacts blend were compressed using tablet compression machine.(Rimek, Karnavati Engineering,India) using flat-faced punch with a compression force thatprovide acceptable tablet hardness.

\section{QbD-DoE approach for $3^{2}$ full factorial design formulations}

On the basis of preliminary studies a $3^{2}$ full factorial design was used to determine the effects of independent variables $\mathrm{X}_{1}$ (Concentration of Chlorthalidone in nonvolatile liquid PEG-400) and $\mathrm{X}_{2}$ (Carrier: coating ratio) on dependent variablesDisintegration time, \% release of drug after 15 minute and 30 minutes. The selection of dependent and independent variables is given in Table-3.

\section{$\mathrm{Y}=\mathrm{b} 0+\mathrm{b} 1 \mathrm{X} 1+\mathrm{b} 2 \mathrm{X} 2+\mathrm{b} 12 \mathrm{X} 1 \mathrm{X} 2+\mathrm{b} 11 \mathrm{X} 12+$ b22X22}

Where $Y$ is the measured response associated with each factor level combination; to intercept b0; regression coefficients b1 and b2 are computed from the observed experimental values of $Y$; and $X 1$ and $X 2$ are the coded level of independent variables. The terms $X 1$ and $\mathrm{X} 2$ represent the interaction and quadratic terms respectively. The selected independent and dependent variables are shown in table along with their low $(-1)$, medium $(0)$ and high $(+1)$ level, which were selected based on the results from preliminary experimentation and literature survey. A design matrix comprising of 9 experimental runs was constructed as shown in table. The concentration of drug in liquid (X1) and carrier: coating ratio (X2) is used to prepare the 9 formulations according to the experimental design.

\section{Precompression Studies Angle of Repose}

The angle of repose of powder blend wasdetermined by fixed height funnel method. Angle of $\operatorname{repose}(\theta)$ was calculated using the following equation:

$$
\theta=\tan ^{-1} \frac{h}{r}
$$

where $h$ and $r$ are the height and radius of powder cone.

\section{Compressibility Index}

The compressibility index of thepowder blendwas determined byCarr's compressibility indexas per equation

$$
\text { Carr's index }=\frac{[(\text { Tapped density }- \text { Bulk density }) \times 100]}{\text { Tapped density }}
$$

\section{Hausner's Ratio}

Hausner's ratio was calculated by following formula

$$
\text { Hausner's ratio }=\frac{\text { Tapped density }}{\text { Bulk density }}
$$

\section{Evaluation of Compressed Tablets Hardness}

The hardness of the tablets was determined Using pharmatest hardness tester. It is expressed in $\mathrm{kg} / \mathrm{cm}^{2}$.

\section{Friability Test}

Roche friabilator (Electrolab) was used for friability test of tablets. 


\section{Disintegration Time}

The disintegration time of the tablets was measured in distilled water $\left(37 \pm 2{ }^{\circ} \mathrm{C}\right)$ using disintegration test apparatus (Electrolab, India).

\section{Content Uniformity}

Five tablets were powdered, and $20 \mathrm{mg}$ equivalent weight of Clorthalidone was accurately weighed and transferred into a $100 \mathrm{~mL}$ volumetric flask. Initially, $10 \mathrm{~mL}$ of methanol was added and shaken for $10 \mathrm{~min}$.Then, the volume was made up to $100 \mathrm{~mL}$ with distilled water. The solution in the volumetric flask was filtered, diluted suitably, and analyzed spectrophotometrically at 275 $\mathrm{nm}$ using UV-visible double beam spectrophotometer (UV1800, Shimadzu, Japan).

\section{In-Vitro Drug Release Study}

The in vitro drug releasestudy of the tablets was performed using USP type Ilapparatus at $37^{\circ} \mathrm{C} \pm 0.5^{\circ}$ Cusing distilled water $(900 \mathrm{~mL})$ as a dissolutionmedium and $50 \mathrm{rpm}$. At the predetermined time intervals, $10 \mathrm{~mL}$ samples were withdrawn and replaced with freshdissolution media.Withdrawn samples were filtered througha $0.45 \mu \mathrm{m}$ membrane filter, diluted, and assayed at $275 \mathrm{~nm}$ using a ShimadzuUV-1800 double-beam

spectrophotometer.

\section{RESULTS AND DISCUSSION Drug excipients compatibility study Fourier Transform Infrared Spectroscopy (FT-IR)}

The FT-IR spectra of Chlorthalidone and physical mixture of drug and excipients are shown in Figure-1. The prominent peaks of drugs was obtained at, 1101, 1690, 1713, and $1062 \mathrm{~cm}^{-1}$ and the prominent peaks of Avicel $\mathrm{PH} 102$ and Aerosil were observed at 2884, 1423, 1530 and 1102.51, $1062.60 \mathrm{~cm}^{-1}$ respectively. All the prominent peaks of drug and excipients were present in the IR spectra of physical mixture indicating the physical compatibility between excipients and drug.

\section{Solubility Study of Chlorthalidone}

Solubility data of drug Chlorthalidone in various liquid vehicles is shown inTable1.Chlorthalidone appears to be more soluble in PEG $400(11.65 \pm 0.135 \mathrm{mg} / \mathrm{ml})$ than other liquids. The solubility is an important factor in liquisolid systems, as higher solubility of drug in liquid vehicle can lead to higher dissolution rates since the drug will be more molecularly dispersed and more surface of drug will be exposed to the dissolution media.

\section{Measuring Angle of Slide}

Angle of slide determination isan important step in the formulation of liquisolid tablets. The relationship of angle of slide with corresponding $\varphi$ of Avicel for Aerosil is shown in Figure-2. The $\varphi \mathrm{Ca}$ and $\varphi \mathrm{Co}$ for liquid vehicles were used to calculate $L f$. The $L f$ was then used to decide the optimum amount of carrier and coating materials required to ensure dry-looking, free-flowing and compactible powdered systems. The lowest liquid factor was obtained for Avicel PH 102 shown in Table-2.

\section{Precompression Studies}

Powder flowability is crucial in the industrial production of tablet dosage forms, as a uniform powder stream through hopper confirms uniformity of both tablet weight and drug content. The results of various flow parameters are shown in Table- 4 .

\section{Quality Control Studies}

All prepared tablets complied with the pharmacopoeial required specifications for the weight variationand content uniformity tests. Results of hardness, friability, disintegration time and drug content are represented in Table-4. Hardnesstest showed an average hardness of liquisolid tablets ranging from 4.3 \pm 0.3 to $4.6 \pm 0.4 \mathrm{Kg} / \mathrm{cm}^{2}$. Another measure oftablets strength is friability. Conventional compressed tablets that lose less than $1 \%$ of their weight are generally consideredacceptable.The percentage friability for all formulations wasbelow $1 \%$, indicating that the friability iswithin the prescribedlimits. This indicates acceptable resistance was shown by liquisolid tablets to withstand handling. Disintegration timewas found to be in the range of $21 \pm 1.16$ to $294 \pm 3.03$ seconds for liquisolid preparations intended for immediate drug release characteristics.

\section{In-vitro dissolution}

The results of in vitro percentage of drug released at different time intervals plotted against time to obtain therelease profile shown in Figure-3. All the liquisolid compacts showed higher drug release than the pure drug. The initial rapid drug release from liquisolid formulation may be due to the use of superdisintegrant which causes a faster deaggregation process leaving the liquisolid particles in the aqueous environment

The results showed thatthere was significant difference between the release profile of the pure drug and all the liquisolid compacts. Theenhanced dissolution rates of liquisolid compacts compared to pure drug may be attributed to the fact that, the drug isalready in 
solution in PG, while at the same time, it is carried by the powder particles. Thus, its release is accelerated due to its markedly increased wettability and surface availability tothe dissolution medium. The wettability of the compacts by the dissolution media is one of the proposed mechanismsfor explaining the enhanced dissolution rate from the liquisolid compacts. The release from all batches appeared immediate release. The batches CL4, CL5and CL9 showed $>80 \%$ drug release within 45 minutes. The results revealed that, when $10 \%$ difference of carrier and coating material ratio showed good dissolution of drug. To determine the effect of independent variables on the dissolution, $3^{2}$ factorial design was applied. The observation response for 9 formulation prepared were fit simultaneously to quadratic model using 9.0.2 design expert. The comparative value of $\mathrm{R} 2$, adjusted value $\mathrm{R} 2$, predicted $\mathrm{R} 2$, adequate precision and \% $\mathrm{CV}$ are given by the quadratic model along with regression equation generate for individual response is given below. From the factorial equation (Equation. 9-11), it can be concluded that the enhancement of drug release is dependent on both the factors under consideration. The carrier: coating concentration is positively influencing the solubilization as it provides surface area for dissolution. It can be observed that more is the carrier: coatingratio better is the solubilization. The reasons behind the effect may be the availability of greater effective surface area due to more concentration of carrier. Less coat concentration imparts less hydrophobic characters to dosage form. Drug concentration in the liquid medication shows negative effect on the dissolution property. This may be because of availability of more amount of solvent for better solubilization of drug.

$$
\begin{aligned}
& \mathrm{Y} 1=82.47+34.50 \times 1-139.50 \times 2- \\
& 28.50 \times 12+14.30 \times 11+73.10 \times 22
\end{aligned}
$$

$$
\begin{gathered}
\text { Y2 }=70.12-8.44 \times 1+15.01 \times 2-1.85 \times 12- \\
0.54 \times 11-5.60 \times 22 \\
Y 3=79.68-4.80 \times 1+14.65 \times 2- \\
1.40 \times 12+3.39 \times 11-10.55 \times 22
\end{gathered}
$$

The effect that favours the optimization that was represent in +ve values and the relationship between factor and response represent in -ve values not favours the optimization. Table-7 shows the effect of drug concentration in PEG $400\left(X_{1}\right)$ and carrier: coating material ratio (X2) has the effect onresponse, disintegration time, $\mathrm{t} 15$ and $\mathrm{t} 30$. Thedatain Table-8 demonstrated that $\mathrm{Y} 1$ and Y3 dependent variable were significant at $5 \%$ level of $P$ values were $<0.05$. The predicted value $R 2$ is reasonable agreement with adjusted model discrimination. The \% CV is < $10 \%$ for $\mathrm{Y} 1$ and $\mathrm{Y} 2$ model which shown that model are reproducible.

\section{Comparison of dissolution data of optimized formulation and conventional formulation}

It is shown in figure-5 that the optimized formulation (OCL9) has higher dissolution rate compare to marketed formulation (CLS). The optimized formulation releases $82.59 \%$ drug in 45 minute whereas marketed formulation releases $64.80 \%$ drug after 45 minute. The liquisolid technique brings about a faster dissolution by different proposed mechanisms of solubility enhancement which include phenomenon of drug being in the dissolved or molecularly dispersed state as well as absorption and adsorption of liquid medication in the internal structures of hydrophilic carrier material providing a greater effective surface area required for the mass transfer of drug molecules from adsorbed and absorbed liquid medication phase to the bulk of dissolution medium. The second mechanism is the cosolvency between the non-volatile hydrophilic solvent and water.

\section{CONCLUSION}

The purpose of the present study was to formulate the liquisolid system of Chlorthalidone for better dissolution rate accompanied by acceptable flow and compression characteristics. In this investigation, preformulation study was performed for authentication of drug and determination of drug solubility. Clorthalidone liquisolid tablet CL9 formulated from $30 \% \mathrm{w} / \mathrm{w}$ of PEG to the drug was found to be superior in terms of dissolution properties in comparison with other liquisolid formulations. Finally, it can be concluded that, liquisolid formulation containing Chlorthalidone with Avicel as carrier and Aerosil as coating material is efficient to enhance the drug dissolution rate with acceptable flow and compression characteristics. Thus, liquisolid approach has potential application for formulation research in improvement of dissolution rate of Chlorthalidone. 
Table 1: Study of solubility in non-volatile oil

\begin{tabular}{|c|c|}
\hline Non volatile Solvent & Solubility $(\mathbf{m g} / \mathbf{m l})$ \\
\hline Polyethylene glycol 400 & $11.65 \pm 0.135$ \\
\hline Polyethylene glycol 600 & $6.17 \pm 0.158$ \\
\hline Polyethylene glycol 300 & $4.76 \pm 0.140$ \\
\hline Propylene glycol & $4.49 \pm 0.085$ \\
\hline Tween 80 & $4.84 \pm 0.014$ \\
\hline Tween 20 & $4.10 \pm 0.115$ \\
\hline
\end{tabular}

Table 2: Liquisolid formulation parameters for powder excipients

\begin{tabular}{|c|c|c|}
\hline Powder excipients & $\begin{array}{l}\text { Ф- values } \\
\text { PEG } 400\end{array}$ & $\begin{array}{l}\Psi \text {-number } \\
\text { PEG } 400\end{array}$ \\
\hline Avicel PH102 & 0.005 & 0.242 \\
\hline Aerosil & 3.26 & 0.653 \\
\hline
\end{tabular}

Table 3: Formulation of liquisolid tablets

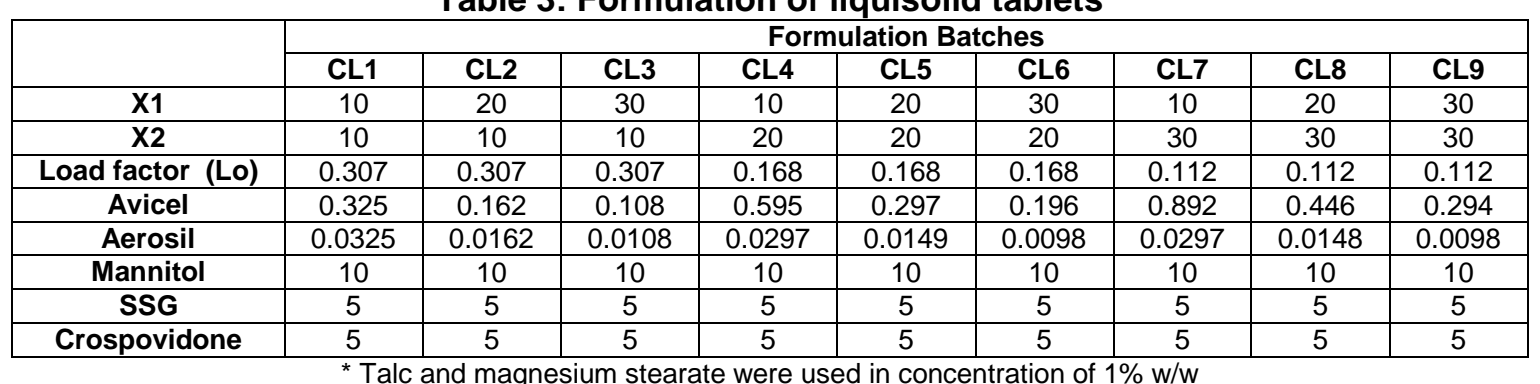

${ }^{* *} \mathrm{X} 1$ Concentration of Chlorthalidone in non-volatile liquid PEG-400 (-1)-10, (0)-(20), (+1)-(30), X2= Carrier: coating ratio ${ }^{* *} \mathrm{Y} 1=$ Disintegration time, $\mathrm{Y} 2=\%$ release of drug after 15 minute, $\mathrm{Y} 3=\%$ release of drug after 30 minute

Table 4: Characterization of powder mixtures

\begin{tabular}{|c|c|c|c|c|c|}
\hline Batches & $\begin{array}{c}\text { Angle of } \\
\text { repose } \\
\left(\mathbf{(}^{\mathbf{)}}\right.\end{array}$ & $\begin{array}{c}\text { Bulk } \\
\text { Density } \\
(\mathbf{g m} / \mathbf{m l})\end{array}$ & $\begin{array}{c}\text { Tapped } \\
\text { density } \\
(\mathbf{g m} / \mathbf{m l})\end{array}$ & $\begin{array}{c}\text { Carr's } \\
\text { Index } \\
(\%)\end{array}$ & Hausner's ratio \\
\hline CL1 & $33.21 \pm 2.0$ & $0.32 \pm 0.08$ & $0.49 \pm 0.09$ & 34.69 & 1.53 \\
\hline CL2 & $32.61 \pm 1.3$ & $0.27 \pm 0.08$ & $0.43 \pm 0.06$ & 37.20 & 1.59 \\
\hline CL3 & $32.0 \pm 0.87$ & $0.34 \pm 0.02$ & $0.52 \pm 0.02$ & 32.69 & 1.48 \\
\hline CL4 & $36.75 \pm 0.9$ & $0.50 \pm 0.07$ & $0.71 \pm 0.06$ & 29.57 & 1.42 \\
\hline CL5 & $29.68 \pm 1.1$ & $0.41 \pm 0.06$ & $0.52 \pm 0.08$ & 21.15 & 1.26 \\
\hline CL6 & $33.0 \pm 1.00$ & $0.36 \pm 0.02$ & $0.61 \pm 0.06$ & 40.90 & 1.68 \\
\hline CL7 & $20.76 \pm 0.80$ & $0.42 \pm 0.06$ & $0.53 \pm 0.04$ & 20.76 & 1.25 \\
\hline CL8 & $27.9 \pm 1.2$ & $0.39 \pm 0.09$ & $0.57 \pm 0.03$ & 31.57 & 1.46 \\
\hline CL9 & $30.11 \pm 1.1$ & $0.41 \pm 0.06$ & $0.52 \pm 0.02$ & 21.15 & 1.26 \\
\hline
\end{tabular}

Table 5: Physical properties of liquisolid tablets

\begin{tabular}{|c|c|c|c|c|}
\hline Batches & $\begin{array}{c}\text { Disintegration } \\
\text { Time (Sec) }\end{array}$ & $\begin{array}{c}\text { Hardness } \\
\mathbf{( k g / \mathbf { c m } ^ { 2 } )}\end{array}$ & $\begin{array}{c}\text { Friability } \\
\mathbf{( \% )}\end{array}$ & $\begin{array}{c}\text { Drug } \\
\text { content (\%) }\end{array}$ \\
\hline CL1 & $247 \pm 0.84$ & $4.3 \pm 0.3$ & 0.22 & $97.56 \pm 1.92$ \\
\hline CL2 & $294 \pm 3.03$ & $4.5 \pm 0.4$ & 0.43 & $99.43 \pm 0.53$ \\
\hline CL3 & $372 \pm 2.65$ & $4.4 \pm 0.2$ & 0.47 & $99.31 \pm 1.28$ \\
\hline CL4 & $64 \pm 1.80$ & $4.6 \pm 0.4$ & 0.30 & $99.8 \pm 1.26$ \\
\hline CL5 & $75 \pm 2.01$ & $4.5 \pm 0.3$ & 0.44 & $98.00 \pm 2.02$ \\
\hline CL6 & $136 \pm 2.08$ & $4.5 \pm 0.4$ & 0.80 & $97.26 \pm 1.71$ \\
\hline CL7 & $24 \pm 3.68$ & $4.4 \pm 0.4$ & 0.91 & $99.4 \pm 0.63$ \\
\hline CL8 & $31 \pm 2.37$ & $4.6 \pm 0.4$ & 0.58 & $97.82 \pm 2.03$ \\
\hline CL9 & $21 \pm 1.16$ & $4.4 \pm 0.4$ & 0.30 & $98.54 \pm 0.78$ \\
\hline
\end{tabular}


Table 6: $3^{2}$ design layout with respective observed responses

\begin{tabular}{|c|c|c|c|c|c|}
\hline Batches & $\begin{array}{c}\mathbf{X 1} \\
\text { drug } \\
\text { concentration } \\
\text { in PEG-400 }\end{array}$ & $\begin{array}{c}\text { X2 } \\
\text { Carrier: } \\
\text { Coating } \\
\text { ratio }\end{array}$ & $\begin{array}{c}\text { Y1 } \\
\text { Disintegration } \\
\text { time } \\
\text { (sec) }\end{array}$ & $\begin{array}{c}\text { Y2 } \\
\text { (drug } \\
\text { release at } \\
\text { 15min) (\%) }\end{array}$ & $\begin{array}{c}\text { Y3 } \\
\text { (drug } \\
\text { release at } \\
\text { 30min) (\%) }\end{array}$ \\
\hline CL1 & -1 & -1 & 247 & 44.19 & 54.73 \\
\hline CL2 & 0 & -1 & 294 & 63.72 & 71.46 \\
\hline CL3 & +1 & -1 & 372 & 48.02 & 55.51 \\
\hline CL4 & -1 & 0 & 64 & 59.85 & 79.2 \\
\hline CL5 & 0 & 0 & 75 & 61.00 & 72.00 \\
\hline CL6 & +1 & 0 & 136 & 47.52 & 55.49 \\
\hline CL7 & -1 & +1 & 24 & 49.95 & 63.40 \\
\hline CL8 & 0 & +1 & 31 & 47.52 & 58.49 \\
\hline CL9 & +1 & +1 & 21 & 68.40 & 77.80 \\
\hline
\end{tabular}

Table 7: Summary of results

\begin{tabular}{|c|c|c|c|c|c|c|c|}
\hline $\mathbf{Y}$ & $\begin{array}{c}\mathbf{P} \\
\text { value }\end{array}$ & $\mathbf{R}^{2}$ & $\begin{array}{c}\text { Adjusted } \\
\mathbf{R}^{2}\end{array}$ & Predicted $\mathbf{R}^{2}$ & $\begin{array}{c}\text { Adequate } \\
\text { Precision }\end{array}$ & $\% \mathbf{C V}$ & Press \\
\hline Y1 & 0.0002 & 0.9985 & 0.9961 & 0.9863 & 52.88 & 5.86 & 1894.28 \\
\hline Y2 & 0.0733 & 0.9185 & 0.7826 & 0.0157 & 7.751 & 11.22 & 1989.30 \\
\hline Y3 & 0.9927 & 0.9927 & 0.9805 & 0.9246 & 23.47 & 2.71 & 127.48 \\
\hline
\end{tabular}

Table 8: results of multiple $\mathrm{Y} 1, \mathrm{Y} 2$ and $\mathrm{Y} 3$

\begin{tabular}{|c|c|c|c|c|c|c|}
\hline \multirow{2}{*}{$\begin{array}{c}\text { Dependent } \\
\text { variables }\end{array}$} & \multicolumn{2}{|c|}{$\begin{array}{c}\text { Y1 = Disintegration } \\
\text { time, } \mathbf{R}^{\mathbf{2}}=\mathbf{0 . 9 9 8 5}\end{array}$} & \multicolumn{2}{c|}{ Y2 = t15 $\mathbf{~ m i n}, \mathbf{R}^{\mathbf{2}}=\mathbf{0 . 9 1 8 5}$} & \multicolumn{2}{c|}{ Y3= t30 $\mathbf{\text { min, }} \mathbf{R}^{\mathbf{2}}=\mathbf{0 . 9 9 2 7}$} \\
\cline { 2 - 7 } & $\mathbf{P}$ value & Coefficients & P value & Coefficients & P value & Coefficients \\
\hline Intercept & 0.0002 & 82.47 & 0.0733 & 70.12 & 0.0021 & 79.68 \\
\hline $\mathrm{X} 1$ & 0.0020 & 34.50 & 0.0685 & 8.44 & 0.0102 & 4.80 \\
\hline $\mathrm{X} 2$ & 0.0001 & 139.50 & 0.0157 & 15.01 & 0.0004 & 14.65 \\
\hline $\mathrm{X} 12$ & 0.0062 & 28.50 & 0.6519 & 1.85 & 0.2613 & 1.40 \\
\hline $\mathrm{X} 11$ & 0.0916 & 14.30 & 0.9244 & 0.54 & 0.0994 & 3.39 \\
\hline $\mathrm{X} 22$ & 0.0011 & 73.10 & 0.3632 & 5.60 & 0.0052 & 10.55 \\
\hline
\end{tabular}

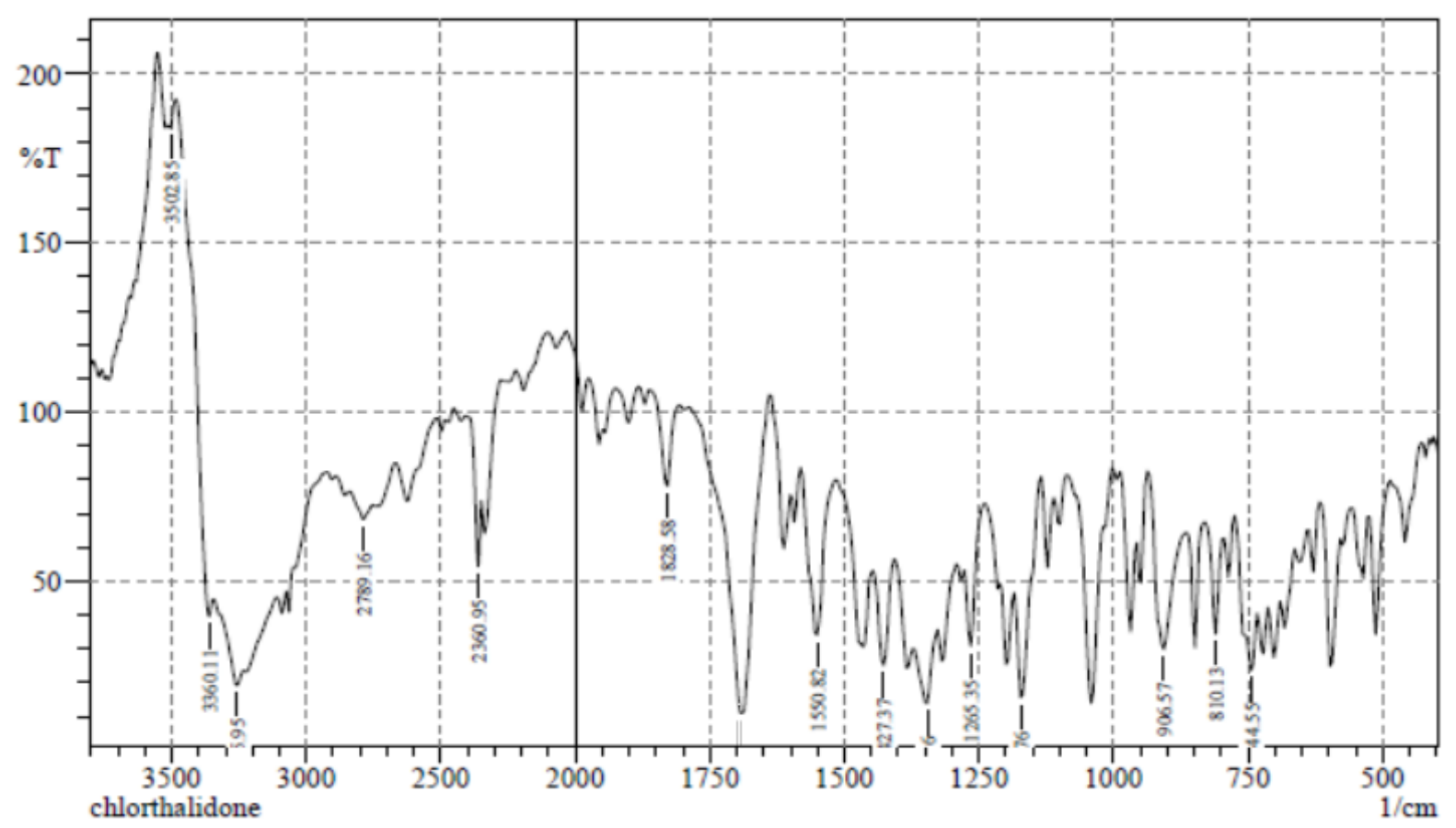

Fig. 1A: IR spectrum of Chlorthalidone (pure drug) 


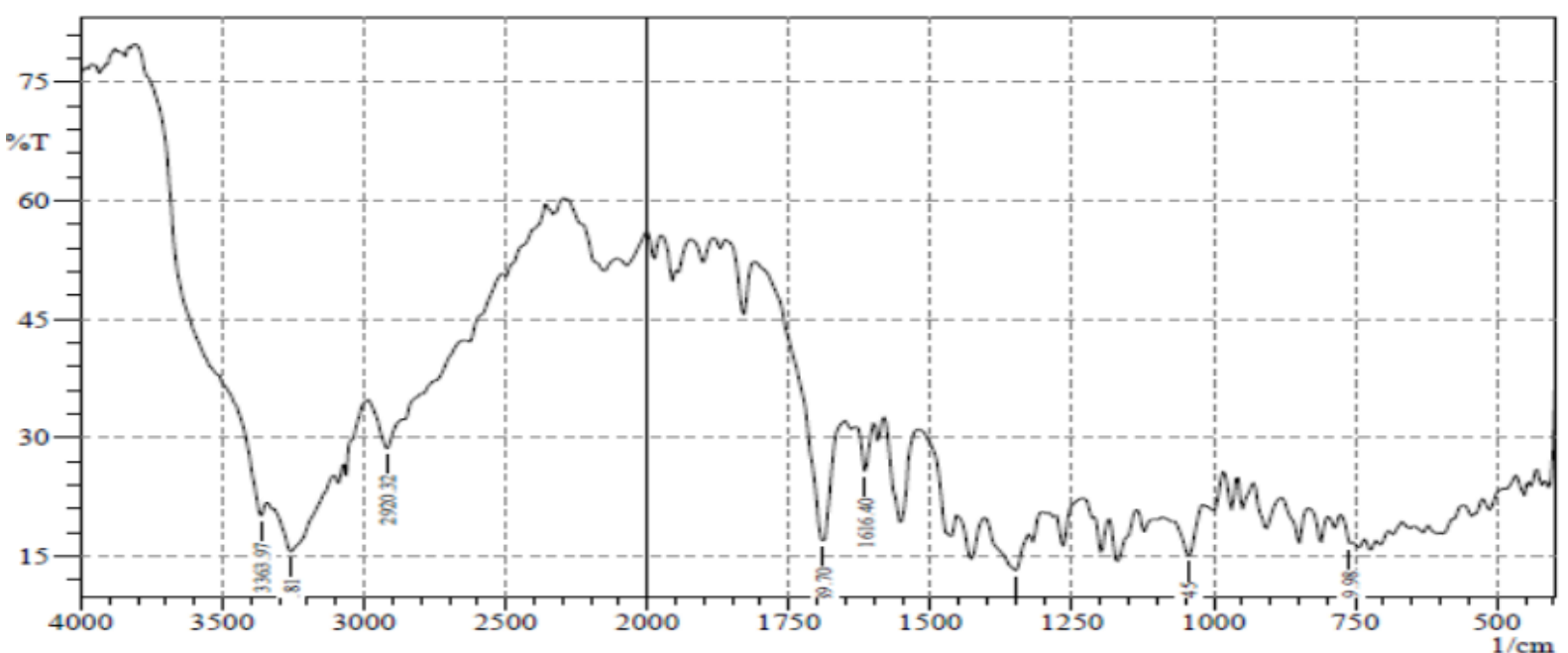

Fig. 1B: IR spectrum of physical mixture of Chlorthalidone and excipients

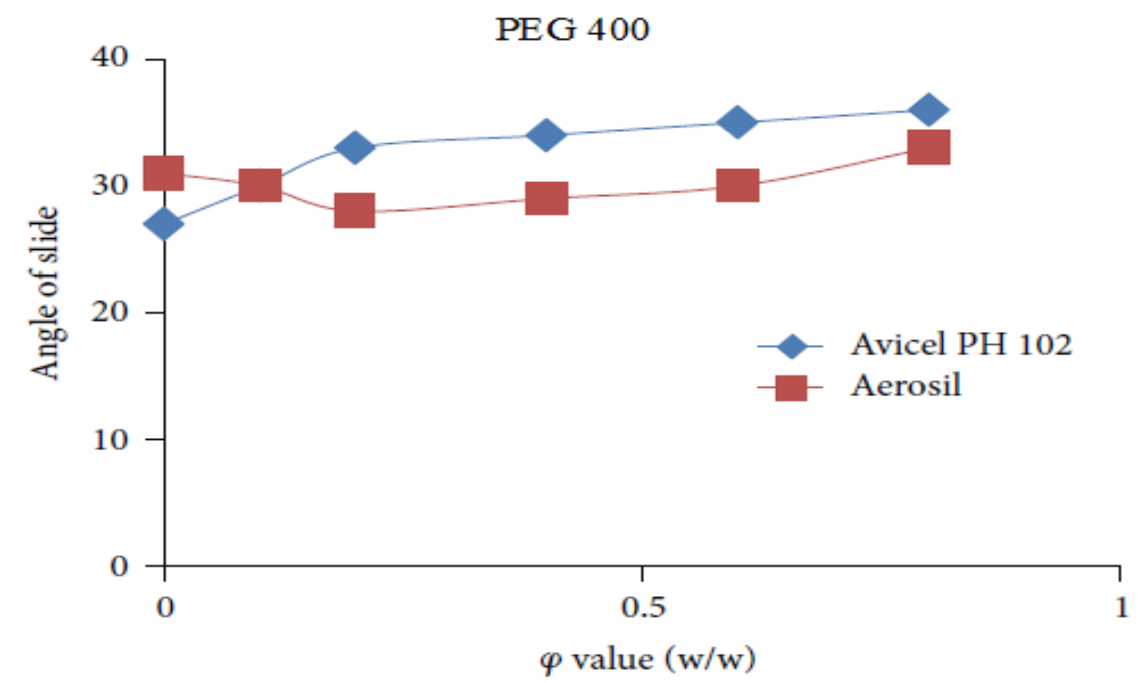

Fig. 2: The angle of slide of Avicel and Aerosil with PEG 400

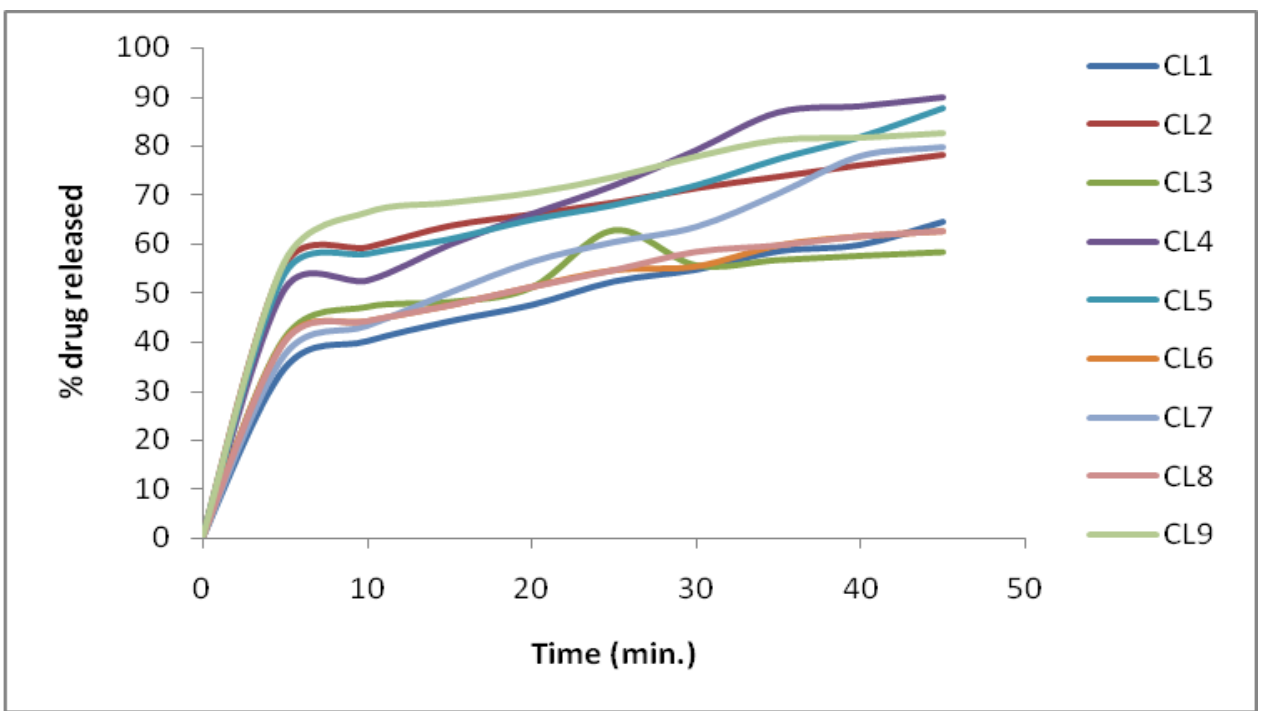

Fig. 3: In-vitro drug release profile 


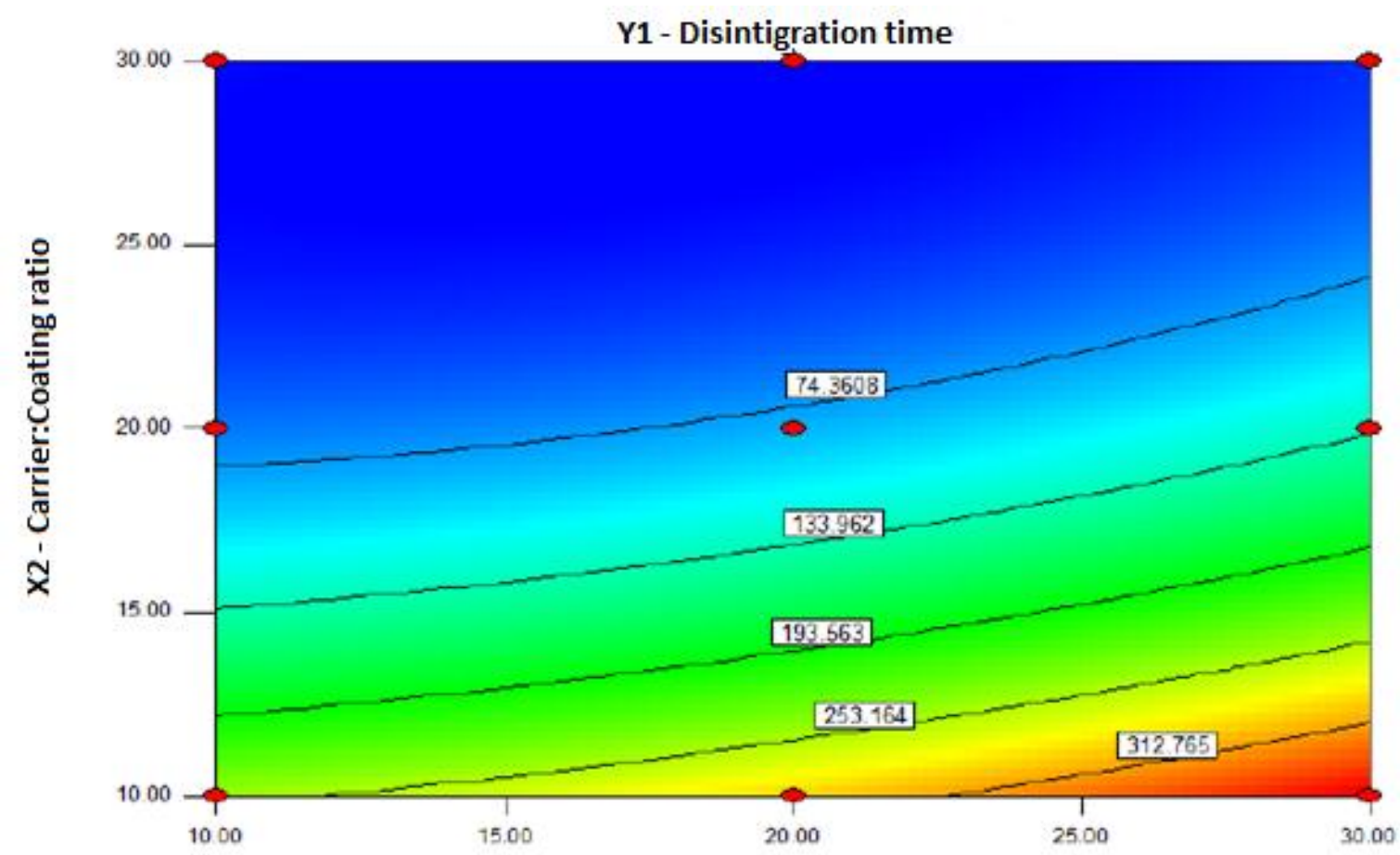

X1 - Concentration of Drug in PEG

A

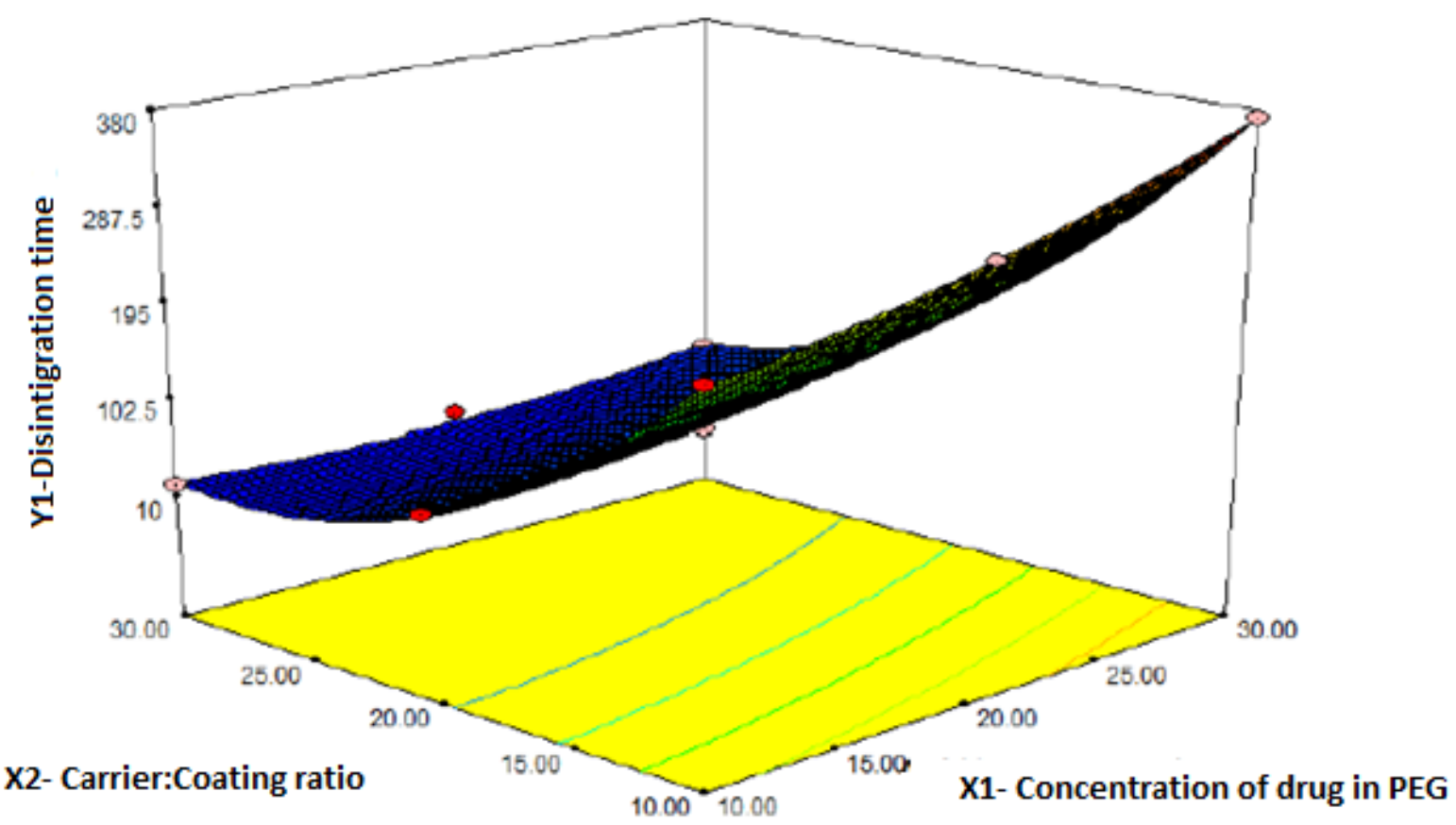

$B$

Fig. 4: (A)-Contour plot and (B) 3-D response surface plot for Disintegration time $\mathrm{Y} 1$ 


\section{Comparison of OCL and Conventional formulation}

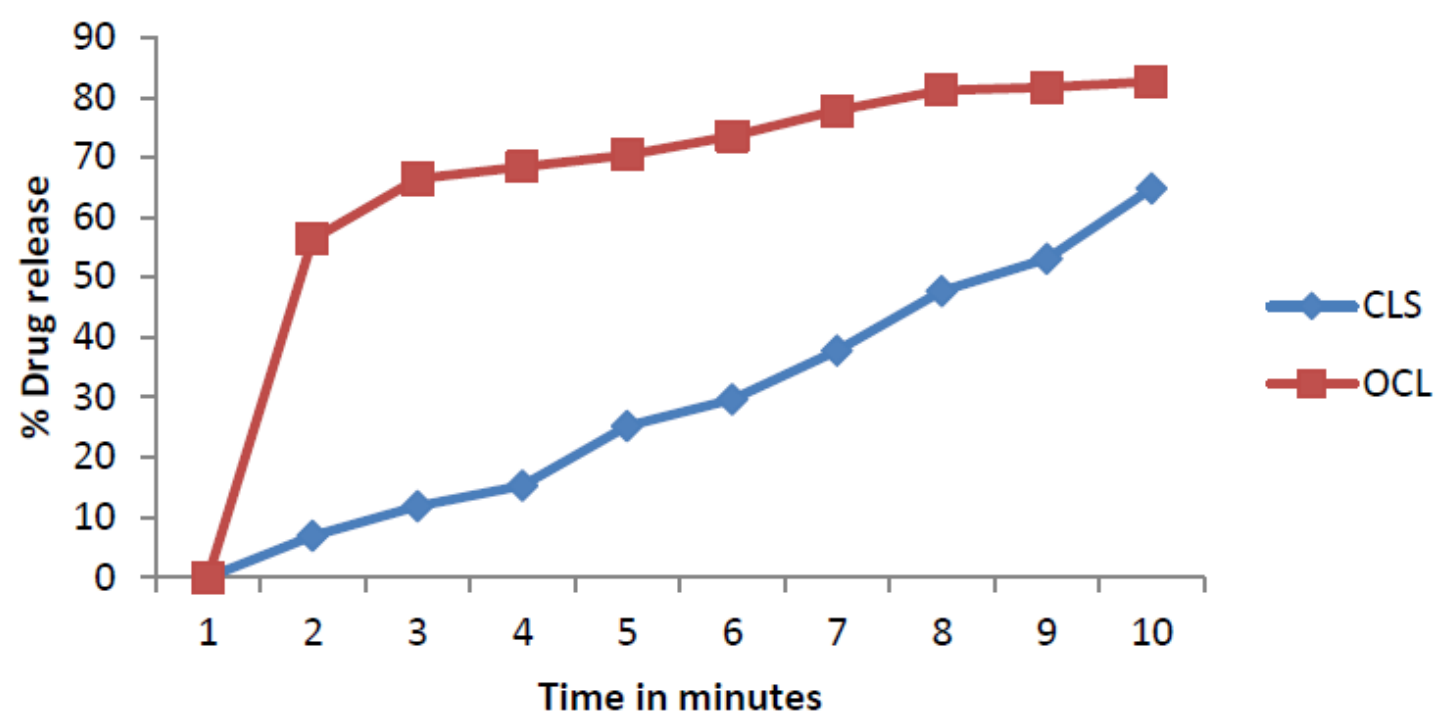

Fig. 5: In-vitro release profile comparison between OCL and conventional tablet

\section{REFERENCES}

1. Patravale VB, Date $A A$ and Kulkarni RM. Nanosuspensions. A Promising Drug Delivery Strategy. J Pharm Pharmacol. 2004;56:827-840.

2. GavaliSahil M, PacharaneSharad $S$ and JadhavKisan R. Liquisolid compact: A new technique for enhancement of drug dissolution. International Journal of research in Pharmacy and Chemistry. 2011;1(3):705-713.

3. Gubbi Sanjeev and Jarag Ravindra. Liquisolid Technique for enhancement of dissolution properties of Bromhexine Hydrochloride. Research J Pharm and Tech. 2009;2(2):382386.

4. Javadzadeh $Y$ and Nokhodchi A. Liquisolid technique for dissolution rate enhancement of a high dose water-insoluble drug (carbamazepine). International Journal of Pharmaceutics. 2007;341:26-34.

5. Savic R, Eisenberg $A$ and Maysinger D. Block Copolymer Micelles as Delivery Vehicles of Hydrophobic Drugs. Micelle-cell interactions. J Drug Target. 2006;14:343-355.

6. Ahuja N, Katare OP and Singh B. Studies on dissolution enhancement and mathematical modeling of drug release of a poorly water-soluble drug using water-soluble carriers. Eur $\mathrm{J}$ Pharm Biopharm. 2007;65:26-38.

7. Spireas $S$ and Sadu S. Enhancement of prednisolone dissolution properties using liquisolid compacts. Int J Pharm. 1998;166:177-188.

8. KarmarkarAmrit B and Gonjarilndrajeet D. Dissolution rate enhancement of Fenofibrate using Liquisolid Tablet Technique, Latin American Journal of Pharmacy. 2009;28(2):219-225.

9. Sanjeev Raghavendra $G$ and Jarag Ravindra. Formulation and Characterization of Atorvastatin Calcium liquisolid compacts. Asian Journal of Pharmaceutical Sciences. 2010;5(2):50-60.

10. Fahmy $\mathrm{RH}$ and Kassem MA. Enhancement of famotidine dissolution rate through liquisolid tablets formulation: In vitro and in vivo evaluation. Eur J Pharm Biopharm. 2008;69:993-1003.

11. Javadzadeh $Y$, Siahi-Shadbad MR, Barzegar-Jalali $M$ and Nokhodchi $A$. Enhancement of dissolution rate of piroxicam compacts. Farmaco. 2005;60:361-336.

12. Saeedi Majid and Akbari Jafar. Enhancement of dissolution rate of Indomethacin using liquisolid compacts. Iranian Journal of 
Pharmaceutical 2011;10(1):25-34

13. Khalid M El-Say, Ahmed M Samy and Mohamed I Fetouh. Formulation and evaluation of Rofecoxibliquisolid tablets. International Journal of Pharmaceutical Sciences Review and Research. 2010;1(3):1-6.

14. Dinesh P, Umesh MS, Mathur D, Vijay $B$ and Bhusari KP. Liquisolid Technique for enhancement of dissolution properties of Carvedilol. Der Pharmacia Letter. 2010;2(15):412427.

15. Santosh $P$, Aparna $C$, Srinivas $P$ and Sadanandam M. Enhancement of dissolution of irbesartan using liquisolid technology. International Journal of Pharmacy and Technology. 2012;4(1):3811-3824.
16. Javadzadeh $Y$, Musaalrezaei $L$ and Nokhodchi Ali. Liquisolid technique as a new approach to sustain propranolol hydrochloride release from tablet matrices. International Journal of Pharmaceutics. 2008;362:102-108.

17. Debnath M, Kumar A and Gopavarapu L. Formulation, Development and InVitro Release Kinetics of Telmisartan Tablet Prepared By Liquisolid Technique. Journal of Pharmaceutical Sciences. 2015;4(3):36-50.

18. Elkordy AA, Essa EA, Dhuppad $S$ and Jammigumpula $P$. Liquisolid technique to enhance and to sustain griseofulvin dissolution. Effect of choice of nonvolatile liquid vehicles. International Journal of Pharmaceutics. 2012;(434)1-2:112-132. 